\title{
Non-local heat flux application for scrape-off layer plasma
}

\author{
H. Bufferand ${ }^{1^{*}}$ | G. Ciraolo ${ }^{1}$ | P. Di Cintio ${ }^{2}$ | N. Fedorczak ${ }^{1}$ | Ph. Ghendrih ${ }^{1} \mid$ S. Lepri $^{2}$ | \\ R. Livi' ${ }^{2,3} \mid$ Y. Marandet ${ }^{4} \mid$ E. Serre ${ }^{5} \mid$ P. Tamain ${ }^{1}$
}

${ }^{1}$ IRFM-CEA, Saint-Paul-Lez-Durance, France

${ }^{2}$ Consiglio Nazionale delle Ricerche, Istituto dei

Sistemi Complessi, Sesto Fiorentino, Italy

${ }^{3}$ Dipartimento di Fisica e Astronomia, Università

di Firenze, Sesto Fiorentino, Italy

${ }^{4}$ Aix-Marseille Université, CNRS, PIIM,

Marseille, France

${ }^{5}$ Aix-Marseille Université, CNRS, M2P2,

Marseille, France

*Correspondence

H. Bufferand, IRFM-CEA, F-13108

Saint-Paul-Lez-Durance, France.

Email: hugo.bufferand@cea.fr

Funding Information

This research was supported by the Euratom,

633053.
The non-local expression proposed by Luciani-Mora-Virmont is implemented in a one dimensional fluid model for the scrape-off layer. Analytical solutions for heat equation are discussed as well as the impact of sheath boundary conditions on the continuity of the temperature profile. The non-local heat flux is compared to the Spitzer-Härm heat flux for different collisionality.

KEYWORDS

heat conduction, non-local transport, plasma physics

\section{1 | INTRODUCTION}

At the entrance of the scrape-off layer, the plasma collisionality $v^{\star}$ defined as the ratio between the field line length and collision mean free path is found to be of order unity. Despite this low collisionality, the tokamak edge plasma modelling relies mostly on the fluid approach and collisional closures that are theoretically only valid at high collisionality. Departure between Braginskii fluid description and kinetic modelling has been highlighted, particularly an underestimation of temperature gradient by the fluid approach. ${ }^{[1]}$ Several kinetic corrections have been proposed to improve the plasma description at intermediate collisionality. ${ }^{[2-5]}$ In this contribution, we investigate kinetic corrections to the local Spitzer-Härm (Braginskii) closure for the heat flux; more precisely, we focus on applying the non-local expression for the heat flux proposed by Luciani-Mora-Virmont ${ }^{[6]}$ to scrape-off layer physics. In particular, we adapt boundary conditions and implement the non-local expression into a 1D hydrodynamic model for the scrape-off layer.

\section{2 | NON-LOCAL FORMULATION FOR HEAT FLUX}

In order to introduce long-range features to the heat flux, in this contribution, we plan to use the non-local expression for the heat flux expressed in Equation (1). This formulation has been proposed in a broad range of references, including Refs. [1, 6, 7].

$$
q_{N L}(x)=\int q_{S H}\left(x^{\prime}\right) w\left(x, x^{\prime}\right) d x^{\prime},
$$

where $q_{S H}$ denotes the Spitzer-Härm expression for the heat flux:

$$
q_{S H}(x)=-\kappa(x) \nabla_{\|} T(x)=-\kappa_{0} T(x)^{5 / 2} \nabla_{\|} T(x)
$$

and $w\left(x, x^{\prime}\right)$ is a kernel describing the space correlation between the temperature gradient and the heat flux. Here, the kernel is an exponential decay, Equation (3), where the decay length is given by the collision mean free path.

$$
w\left(x, x^{\prime}\right)=\frac{1}{2 \lambda\left(x^{\prime}\right)} \exp \left(-\frac{\left|\int_{x^{\prime}}^{x} n\left(x^{\prime \prime}\right) d x^{\prime \prime}\right|}{n\left(x^{\prime}\right) \lambda\left(x^{\prime}\right)}\right) .
$$


This kernel tends to a Dirac centred on $x$ for high collisionality, that is, when $\lambda \rightarrow 0$. In this case, one recovers the local Fourier law. For flat density profiles and neglecting the temperature dependence upon the mean free path, the kernel $w\left(x, x^{\prime}\right)$ reduces to

$$
\tilde{w}\left(x, x^{\prime}\right)=\frac{1}{2 \lambda} \exp \left(-\frac{\left|x-x^{\prime}\right|}{\lambda}\right) .
$$

In this case, the non-local heat flux given by Equation (1) can be re-written in the form of a convolution product of Spitzer-Härm heat flux and an exponential kernel; see Equation (5).

$$
\tilde{q}_{N L}(x)=\int \frac{1}{2 \lambda} \exp \left(-\frac{\left|x-x^{\prime}\right|}{\lambda}\right) q_{S H}\left(x^{\prime}\right) d x^{\prime}=\left(\tilde{w}^{*} q_{S H}\right)(x) .
$$

In order to emphasize the behaviour of such a non-local expression for the heat flux, let us compute analytical solutions for the heat equation $\partial_{x} \tilde{q}_{N L}=S$ in a periodic 1D domain. We solve the equation in the Fourier space, $q(x)=1 / 2 \pi \int_{-\infty}^{\infty} \hat{q}(k) e^{i k x}$, the Fourier transform of the convolution product being the product of the Fourier transforms:

$$
\begin{aligned}
i k \mathscr{F}\left[\tilde{q}_{N L}\right] & =\mathscr{F}[S], \\
\kappa k^{2} \mathscr{F}[\tilde{w}] \mathscr{F}[T] & =\mathscr{F}[S],
\end{aligned}
$$

where we neglected the dependence of the temperature on $\kappa \cdot{ }^{1}$ The Fourier transform of the exponential kernel is given by a Lorentzian:

$$
\mathscr{F}[\tilde{w}]=\frac{1}{1+\lambda^{2} k^{2}} .
$$

Reporting in Equation (6) gives

$$
\mathscr{F}[T]=\left(\frac{1}{\kappa k^{2}}+\frac{\lambda^{2}}{\kappa}\right) \mathscr{F}[S]
$$

Back to the real space, one finds

$$
T(x)=T(0)+x \cdot\left(\partial_{x} T(0)-\frac{\lambda^{2}}{\kappa} \partial_{x} S(0)\right)-\left(\frac{1}{\kappa} \int_{0}^{x} d x^{\prime} \int_{0}^{x^{\prime}} d x^{\prime \prime} S\left(x^{\prime \prime}\right)\right)+\frac{\lambda^{2}}{\kappa} S(x) .
$$

The temperature profiles are thus obtained by summing the double integration of the source (high collisional diffusive behaviour) and the source itself ponderated by $\lambda^{2} / \kappa$. The last term can be neglected at high collisionality. At low collisionality, when $\lambda$ is not zero, the shape of the source is recovered in the temperature profile, and if the source is not continuous (e.g., punctual sources modelled as Dirac distributions), neither is the temperature profile. Figure 1 shows temperature profiles for punctual source and sink $S(x)=\delta\left(x-x_{h}\right)-\delta\left(x-x_{c}\right)$ for different values of the collisionality. At low collisionality, the temperature profile is continuous. An analytical solution is also found with continuous Gaussian sources. In this case, the solution is continuous at any collisionality. From this analytical analysis, one finds that the non-local formulation for the heat flux does not guarantee the continuity of the temperature profile. However, as long as the source is continuous (which is the case in most applications), so is the temperature profile. What about the continuity when adding non-periodic boundary conditions, which is necessary for an application to the plasma scrape-off layer?

\section{3 | BOUNDARY CONDITIONS}

\section{1 | Heat equation with Dirichlet boundary conditions}

In order to study the continuity of the temperature profile for a tokamak SOL application, let us consider first a simple application to the non-local heat flux expression to a finite 1D domain of length $L_{\|}$with Dirichlet boundary conditions:

$$
\left\{\begin{array}{l}
\partial_{x} \tilde{q}_{N L}=0, \\
T(0)=T_{\text {hot }} \quad \text { and } \quad T\left(L_{\|}\right)=T_{\text {cold }} .
\end{array}\right.
$$

Assuming a constant temperature gradient $\left(\partial_{x} T=\alpha\right)$ and reporting in the non-local heat flux expression, one finds:

$$
\begin{aligned}
\tilde{q}_{N L}(x) & =-\int_{0}^{L_{\|}} \frac{1}{2 \lambda} \exp \left(-\frac{\left|x-x^{\prime}\right|}{\lambda}\right) \kappa \alpha d x^{\prime} \\
& =\frac{\alpha \kappa}{2}\left[\exp \left(-\frac{x}{\lambda}\right)+\exp \left(-\frac{L_{\|}-x}{\lambda}\right)-2\right] .
\end{aligned}
$$


(a)

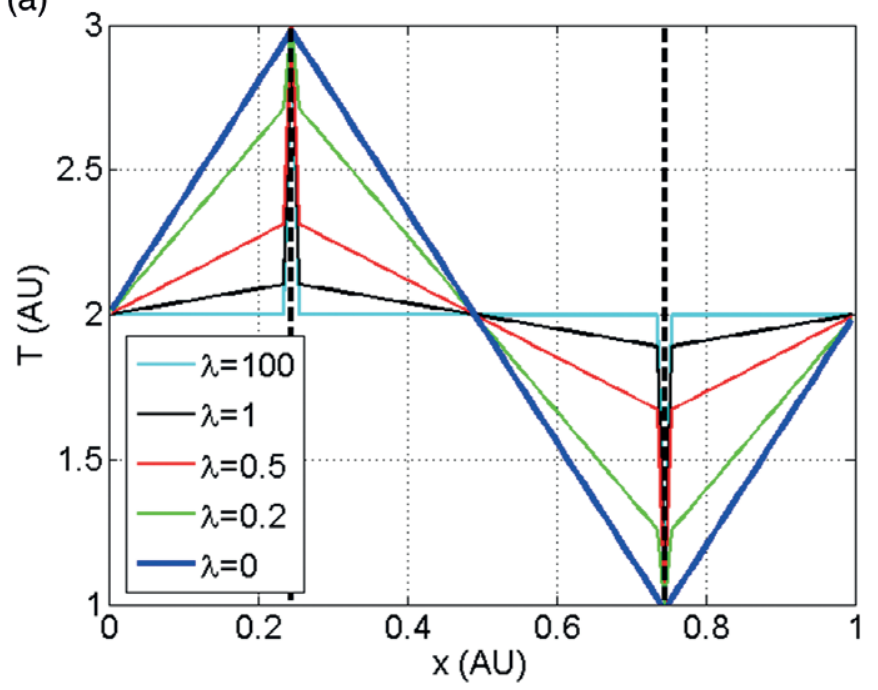

(b)

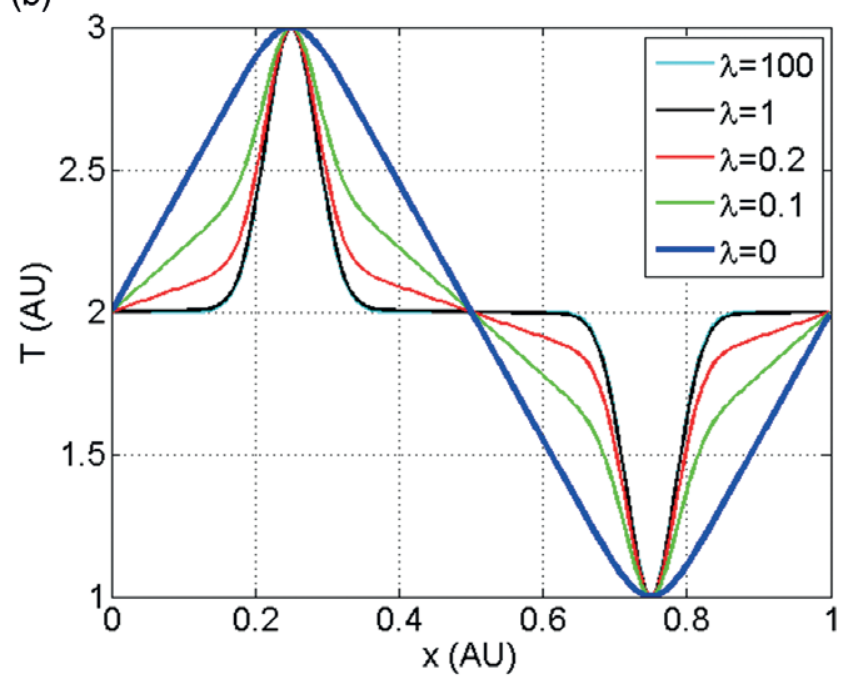

FIGURE 1 Temperature profiles obtained by solving a heat equation with simplified non-local heat flux expression and punctual (a) and Gaussian (b) heat source and sink

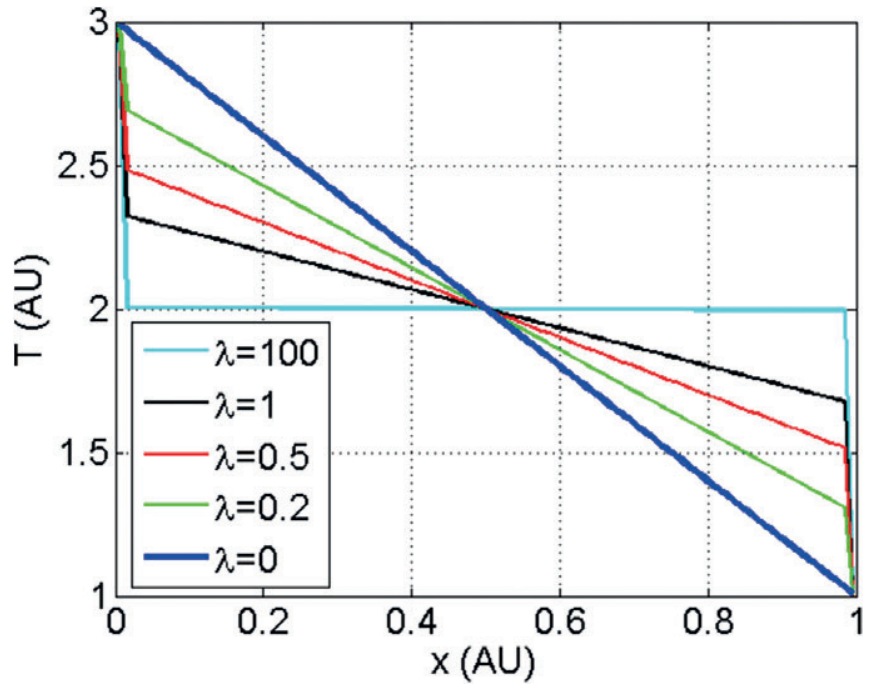

FIGURE 2 Analytical solution to the heat equation with the simplified non-local expression for the heat flux in a finite 1D domain with Dirichlet boundary conditions. The solutions are plotted for different values of the mean free path $\lambda$

In order to obtain a constant heat flux consistant with $\partial_{x} q=0$, one needs to complete the temperature gradient so as to cancel the two exponential functions in Equation (11). This is made possible by adding Dirac functions:

$$
\partial_{x} T=\alpha\left(1+\lambda \delta_{0}(x)+\lambda \delta_{L_{\|}}(x)\right)
$$

One finds the expression $q=-\alpha \kappa$ with this temperature gradient. The temperature is obtained by integrating Equation (12) and considering boundary conditions:

$$
T(x)=T_{\mathrm{hot}}+\alpha\left(x+\lambda H(x)+\lambda H\left(x-L_{\|}\right)\right),
$$

where $H(x)$ denotes the Heaviside function. The value of $\alpha$ is found by evaluating Equation (13) in $L_{\|}$:

$$
\alpha=\frac{T_{\mathrm{cold}}-T_{\mathrm{hot}}}{L_{\|}+2 \lambda} .
$$

Solutions are plotted for different values of $\lambda$ on Figure 2 . As with the punctual sources, one finds once again that the temperature profile is not continuous. The discontinuity appears this time at the domain boundary. In order to use the non-local kernel combined with a hydrodynamic approach of the plasma, one needs to guarantee the continuity of the temperature, including at the boundary. In the following paragraph, we propose a practical way to treat the sheath boundary condition at the ends of the magnetic field line in order to guarantee the continuity of the temperature profile. 


\subsection{Non-local boundary conditions for the sheath}

From the previous section, we see that the use of non-local expression for the heat flux may lead to discontinuities in the temperature profile at the domain boundary. The temperature can be decomposed as $T=\tilde{T}+T_{B C}$ where $\tilde{T}$ is continuous; by definition, we impose $\partial_{x} \tilde{T}(0)=\partial_{x} \tilde{T}\left(L_{\|}\right)=0$. The temperature gradient is expressed by

$$
\partial_{x} T=\partial_{x} \tilde{T}+\partial_{x} T_{B C}=\partial_{x} \tilde{T}+\partial_{x} T(0) \delta(x)+\partial_{x} T\left(L_{\|}\right) \delta\left(x-L_{\|}\right) .
$$

Reporting Equation (15) in the non-local expression for the heat flux gives

$$
q_{N L, T}(x)=q_{N L, \tilde{T}}(x)+q_{B C, 0} \exp \left(-\frac{x}{\lambda}\right)+q_{B C, L_{\|}} \exp \left(\frac{x-L_{\|}}{\lambda}\right) .
$$

This expression exhibits a first term describing the non-local heat flux computed from the continuous temperature gradient $\partial_{x} \tilde{T}$ in the plasma. The last two terms represent the impact of the boundary condition in the heat flux, an effect that decays exponentially away from the wall on a typical length given by the collisional mean free path. The values $q_{B C, 0}$ and $q_{B C, L_{\|}}$are adjusted to match the sheath boundary condition for the heat flux, namely, $q_{s e}=\gamma n v_{\|} T$, at both ends where $\gamma$ is the so-called sheath transmission coefficient. Thus, one has the following at both ends of the field line:

$$
\begin{gathered}
\gamma n(0) v_{\|}(0) T(0)=\tilde{q}_{N L, \tilde{T}}(0)+q_{B C, 0}+q_{B C, L_{\|}} \exp \left(-\frac{L_{\|}}{\lambda}\right), \\
\gamma n\left(L_{\|}\right) v_{\|}\left(L_{\|}\right) T\left(L_{\|}\right)=\tilde{q}_{N L, \tilde{T}}\left(L_{\|}\right)+q_{B C, 0} \exp \left(-\frac{L_{\|}}{\lambda}\right)+q_{B C, L_{\|}},
\end{gathered}
$$

that denotes $\nu^{\star}=L_{\|} / \lambda$ and $\beta=\exp \left(-\nu^{\star}\right)$ :

$$
\begin{aligned}
q_{B C, 0} & =\frac{q_{s e}(0)-\tilde{q}_{N L, \tilde{T}}(0)-\beta\left(q_{s e}\left(L_{\|}\right)-\tilde{q}_{N L, \tilde{T}}\left(L_{\|}\right)\right)}{1-\beta^{2}}, \\
q_{B C, L_{\|}} & =\frac{q_{s e}\left(L_{\|}\right)-\tilde{q}_{N L, \tilde{T}}\left(L_{\|}\right)-\beta\left(q_{s e}(0)-\tilde{q}_{N L, \tilde{T}}(0)\right)}{1-\beta^{2}} .
\end{aligned}
$$

\section{4 | APPLICATION TO 1D SOL MODELLING}

The non-local model with the abovementioned sheath boundary condition is applied to simulate a 1D SOL hydrodynamic. The following system of equations is solved for mass, parallel momentum, and ion and electron energy balance:

$$
\left\{\begin{array}{l}
\partial_{t} n+\vec{\nabla} \cdot\left(n v_{\|} \vec{b}\right)=S_{n}, \\
\partial_{t}\left(m_{i} n v_{\|}\right)+\vec{\nabla} \cdot\left(m_{i} n v_{\|}^{2} \vec{b}\right)=-\nabla_{\|} p_{i}+q n E_{\|}, \\
\partial_{t}\left(\frac{3}{2} n T_{i}+\frac{1}{2} m_{i} n v_{\|}^{2}\right)+\vec{\nabla} \cdot\left(\left[\frac{5}{2} n T_{i}+\frac{1}{2} m_{i} v_{\|}^{2}\right] v_{\|} \vec{b}\right)+\vec{\nabla} \cdot\left(q_{i \|} \vec{b}\right)=q n v_{\|} E_{\|}-\frac{3}{2} \frac{n}{\tau_{e i}}\left(T_{i}-T_{e}\right)+S_{E i}, \\
\partial_{t}\left(\frac{3}{2} n T_{e}\right)+\vec{\nabla} \cdot\left(\frac{5}{2} n T_{e} v_{\|} \vec{b}\right)+\vec{\nabla} \cdot\left(q_{e \|} \vec{b}\right)=-e n v_{\|} E_{\|}-\frac{3}{2} \frac{n}{\tau_{e i}}\left(T_{e}-T_{i}\right)+S_{E e},
\end{array}\right.
$$

where $E_{\|}=-\left(\nabla_{\|} p_{e}\right) /(e n)$. The following boundary conditions apply:

$$
\begin{cases}\text { Neumann : } & \nabla_{\|} n(0)=\nabla_{\|} n\left(L_{\|}\right)=0, \\ \text { Bohm : } & v_{\|}(0) \leq-\sqrt{\frac{T_{e}(0)+T_{i}(0)}{m_{i}}} \quad \text { and } \quad v_{\|}\left(L_{\|}\right) \geq \sqrt{\frac{T_{e}\left(L_{\|}\right)+T_{i}\left(L_{\|}\right)}{m_{i}}}, \\ \text { Sheath heat flux }: & q_{i(e) \|}=\left(\gamma_{i(e)}-\frac{5}{2}\right) n T_{i(e)} v_{\|} \quad \text { at } 0 \text { and } L_{\|} \text {location, }\end{cases}
$$

where $\gamma_{i}=2.5$ and $\gamma_{e}=4.5$. First, simulations are run with a fixed and homogeneous particle source $S_{n}(x)=S_{n}^{0}$. The energy source is also taken homogeneous $\left(S_{E e, i}(x)=S_{E e, i}^{0}\right)$, and the intensity of the energy source is scanned to change plasma temperature and thus plasma collisionality. Figure 3 shows electron temperature profiles computed for different collisionalities and using different expressions for the heat flux:

- Spitzer-Härm: $q=q_{S H}=-\kappa_{S H} \nabla_{\|} T$

- Flux-limited Spitzer-Härm: $q=q_{F L}=\left(q_{S H}^{-1}+q_{\max }^{-1}\right)^{-1}$ with $q_{\max }=\alpha m_{e} n c_{e}^{3}$, where $c_{e}=\sqrt{T_{e} / m_{e}}$ and $\alpha=0.15$

- Non-local heat flux $q=q_{N L}$ 
(a)

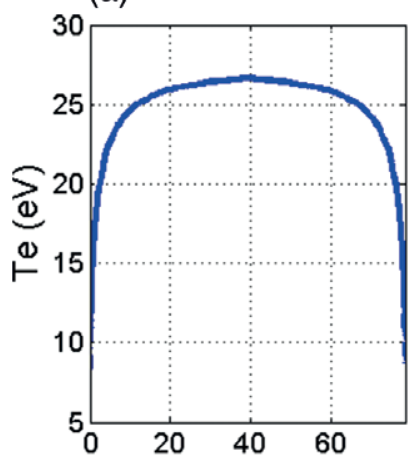

(a)

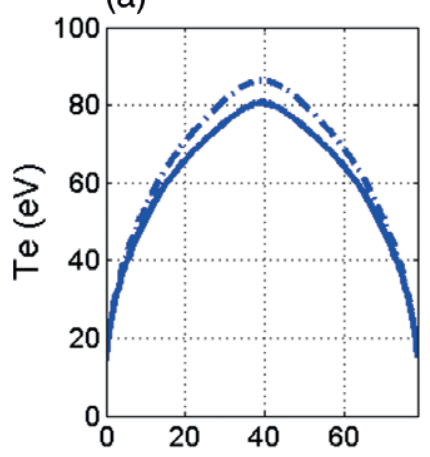

(b)

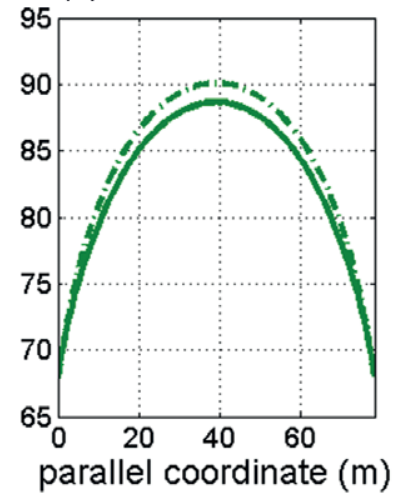

(b)

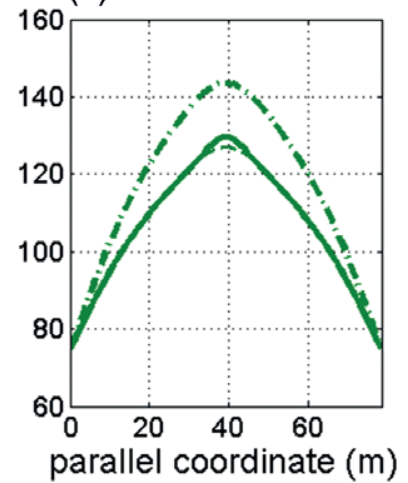

(c)

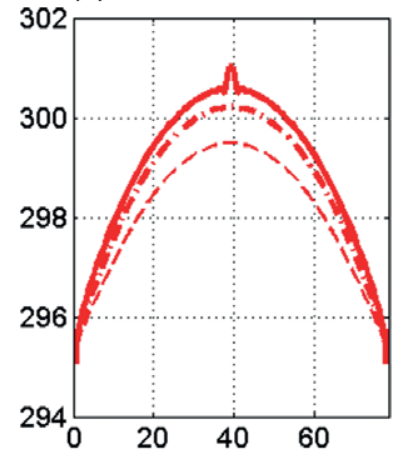

(c)

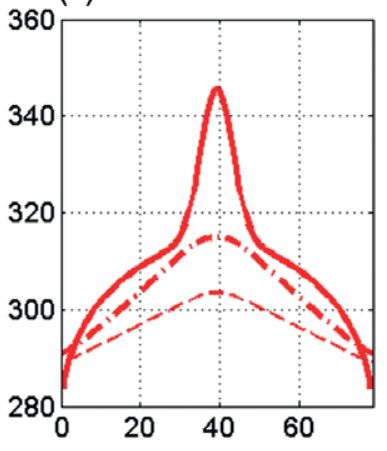

FIGURE 3 Electron temperature profiles for different heat flux expressions (solid line: non-local, dashed line: Spitzer-Härm, dot-dashed line: flux limited SpitzerHärm). The particle and energy sources are homogeneous. (a) High collisionality $\left(v^{\star} \approx 60\right)$, (b) intermediate collisionality $\left(v^{\star} \approx 10\right)$, (c) low collisionality $\left(v^{\star} \approx 1\right)$

FIGURE 4 Electron temperature profiles for different heat flux expressions (solid line: nonlocal, dashed line: Spitzer-Härm, dot-dashed line: flux limited SpitzerHärm). The particle and energy sources are peaked. (a) High collisionality $\left(v^{\star} \approx 60\right)$, (b) intermediate collisionality $\left(v^{\star} \approx 10\right)$, (c) low collisionality $\left(v^{\star} \approx 1\right)$

Unlike the previous sections, in the simulations, the temperature dependence of $T$ on $\kappa_{S H}$ is well considered, both for local and non-local expressions. For the latter, the dependence on $\lambda$ is also taken into account.

To go further and see the impact of the shape of the source, we now consider a particle source located close to the ends of the field line to simulate recycling:

$$
S_{n}(x)=S_{n}^{0}\left[\exp \left(-\frac{x}{0.1 L_{\|}}\right)+\exp \left(-\frac{L_{\|}-x}{0.1 L_{\|}}\right)+0.005\right] .
$$

For the energy sources, we use Gaussian-shaped sources located at the middle of the field line. The width of the energy source is controlled by $\lambda_{E}$.

$$
S_{E e, i}=S_{E e, i}^{0} \exp \left(-\left(\frac{x}{\lambda_{E}}-\frac{L_{\|}}{2 \lambda_{E}}\right)^{2}\right) .
$$

Figure 4 shows simulation results for $\lambda_{E}=0.1 L_{\|}$. Once again, the intensity of the source is ramped to change the collisionality.

\section{5 | DISCUSSION}

In Figures 3 and 4, for high and medium collisonality, there is almost no difference in the temperature profiles obtained considering either the non-local expression or the Spitzer-Härm expression. At medium collisionality, a noticeable effect of the flux limiter leads to increasing temperature gradients for the same heat flux. This leads to an increase of the upstream temperature. Such an increase of the upstream temperature is not observed using the non-local heat flux. Another way to analyse the effect of the non-local expression consists of recalculating the heat fluxes as a post-treatment with the different expressions for a given temperature profile. The left panel of Figure 5 shows electron heat fluxes calculated from the electron temperature profile obtained at high collisionality with the non-local heat flux and with a peaked source. One finds a relatively good agreement between the different heat flux expressions, particularly between Spitzer-Härm and the non-local expression. All heat flux expressions give values much below the maximum heat flux value plotted in red as $q_{\max }=0.15 q_{F S}$ with the free streaming heat flux beginning given by $q_{F S}=n_{e} m_{e} c_{e}^{3}$ with $c_{e}=\sqrt{T_{e} / m_{e}}$.

The situation is more interesting at lower collisionality, particularly with peaked sources; see the right panel of Figure 4. It can be noticed that the temperature profile obtained with the non-local heat flux expression shows the superposition of the shape of the sources (temperature peak in the middle of the field line as well as temperature drop close to the wall due to the 
(a)

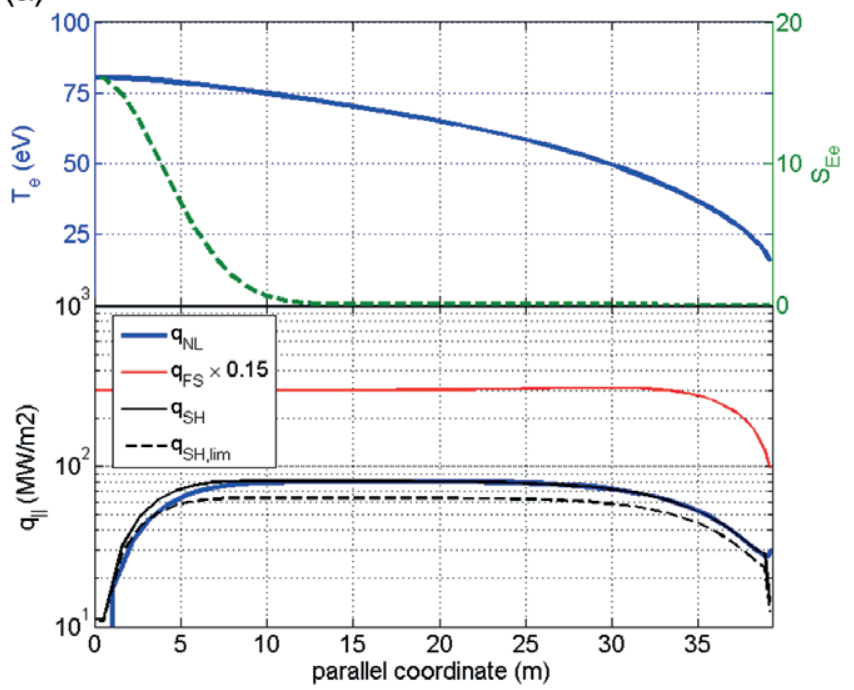

(b)

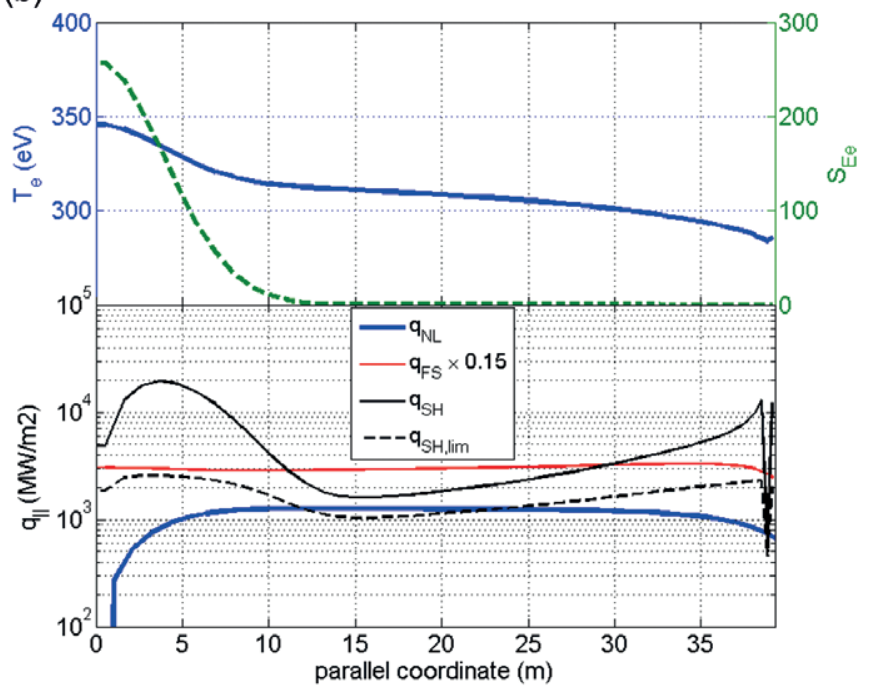

FIGURE 5 Comparison of heat fluxes computed as a post-treatment from the electron temperature profile. (a) High collisionality, (b) low collisionality. Top: temperature and source profiles, bottom: heat fluxes

plasma energy dilution induced by the particle source) on top of a more standard temperature decay that is observed considering diffusive expression for the heat flux. This superposition of the diffusive solution and the source shape was already found analytically in Equation (9).

The right panel of Figure 5 shows heat flux values computed as a post-treatment from the electron temperature profiles obtained with the non-local heat flux expression at low collisionality. Unlike the non-local expression, one notices that the Spitzer-Härm expression gives value way above $q_{\max }$. The non-local heat flux expression thus actively plays as a flux limiter. Likewise, one notices obviously that the flux-limited Spitzer-Härm heat flux takes a value below $q_{\max }$, that is, the purpose of the flux-limited expression. However, when comparing non-local heat flux with local flux-limited Spitzer-Härm, one observes a different shape of heat flux, the non-local expression exhibiting a long-range effect of the strong temperature gradient near the energy source. This effect is reminiscent of what is observed in laser-heated plasma where the heat source is localized. ${ }^{[6]} \mathrm{A}$ localized heat sink near the wall can also drive these strong temperature gradients as observed in Ref. [1].

\section{I CONCLUSIONS AND PERSPECTIVES}

Using non-local heat flux for electron conduction appears to make the recovery of strong temperature gradients and long-range interactions at intermediate collisionality possible. The heat flux is found to be lower than the maximum free-streaming heat flux. Future comparisons with kinetic PIC simulations ${ }^{[(8,9)]}$ should confirm or contradict results obtained with the non-local expression.

\section{ACKNOWLEDGMENTS}

This work was granted access to the HPC resources of Aix-Marseille Universite financed by the project Equip@Meso (ANR-10-EQPX-29-01) of the programme "Investissements d'Avenir" supervised by the Agence Nationale pour la Recherche. This work has been carried out within the framework of the EUROfusion Consortium and has received funding from the Euratom research and training programme 2014-2018 under grant agreement No. 633053 for the project WP17-ENR-CEA-01. The views and opinions expressed herein do not necessarily reflect those of the European Commission.

\section{NOTE}

1. The same algebra could be performed considering the temperature dependence in $\kappa$ by performing the change of variable $T \leftarrow \Theta$ and $\kappa_{0} \leftarrow \frac{2}{7} \kappa_{0}$ where $\Theta=T^{7 / 2}$. 


\section{REFERENCES}

[1] R. Chodura, Contrib. Plasma Phys. 1992, 32, 219.

[2] J. P. Brodrick, R. J. Kingham, M. M. Marinak, M. V. Patel, A. V. Chankin, J. T. Omotani, M. V. Umansky, D. Del Sorbo, B. Dudson, J. T. Parker, G. D. Kerbel, M. Sherlock, C. P. Ridgersn, Phys. Plasma 2017, 24, 092309 (2017), https://doi.org/10.1063/1.5001079.

[3] A. V. Chankin, D. P. Coster, J. Nucl. Mater. 2017, 463, 498-5017 (2015), https://doi.org/10.1016/j.jnucmat.2014.10.057.

[4] Y. L. Igitkhanov, A. M. Runov, Contrib. Plasma Phys. 1992, 32, 308.

[5] A. S. Kukushkin, A. M. Runov, Contrib. Plasma Phys. 1994, 34, 204.

[6] J. F. Luciani, P. Mora, Phys. Rev. Lett. 1983, 51, 1664.

[7] H. Bufferand, G. Ciraolo, P. Ghendrih, S. Lepri, R. Livi, Phys. Rev. E 2013, 87, 023102 (2013), https://doi.org/10.1103/PhysRevE.87.023102.

[8] J. P. Gunn, J. Nucl. Mater. 2005, 337-339, 310.

[9] D. Tskhakaya, Contrib. Plasma Phys. 2016, 56, 698. 